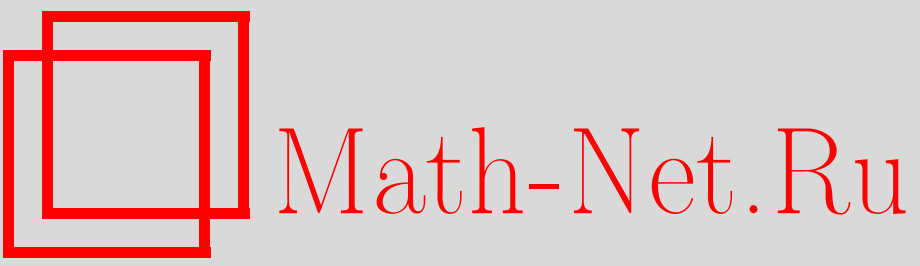

О. В. Шварцман, Свободные $G$-пучки на замкнутых римановых поверхностях, УМH, 1999, том 54, выпуск 6, 175-176

DOI: https://doi.org/10.4213/rm246

Использование Общероссийского математического портала Math-Net.Ru подразумевает, что вы прочитали и согласны с пользовательским соглашением

http://www.mathnet.ru/rus/agreement

Параметры загрузки:

IP: 54.172 .240 .79

26 апреля 2023 г., 08:29:04 


\title{
СВОБОДНЫЕ G-ПУЧКИ НА ЗАМКНУТЫХ РИМАНОВЫХ ПОВЕРХНОСТЯХ
}

\author{
О.В. ШВАРЦМАН
}

1. Определения. Пусть $X$ - замкнутая риманова поверхность, $G$ - такая конечная группа ее автоморфизмов, что $X / G=\mathbb{P}^{1}(\mathbb{C})$. Рассмотрим одномерное комплексное $G$-расслоение $L$ над $X$ (в дальнейшем - обратимьй $G$-пучок). $G$-пучок $L$ назовем эффективным, если единичный элемент группы тождественно действует в пространстве расслоения $L$.

Через $G_{x}$ обозначим стабилизатор точки $x \in X$. $G_{x}$ - это циклическая группа порядка $n_{x}$. Существует такая окрестность $U_{x}$ точки $x$, что действие образующей группы $G_{x}$ в $L \mid U_{x}$ имеет вид

$$
\left(z_{x}, t\right) \mapsto\left(e^{2 \pi \sqrt{-1} / n_{x}} z_{x}, e^{2 \pi \sqrt{-1} r_{x} / n_{x}} t\right), \quad 0 \leqslant r_{x}<n_{x},
$$

где $z_{x}$ - локальньй параметр в точке $x, t \in \mathbb{C}$. Предположим, что НОД $\left(n_{x}, r_{x}\right)=1$, если $r_{x}>0$. Общий случай к этому легко сводится. Пару $\left(n_{x}, r_{x}\right)$ назовем показателем точки $x$. Любые две точки, лежащие на одной орбите группы $G$ в $X$, имеют одинаковый показатель, и для всех $G$-орбит, кроме конечного числа, он равен $(1,0)$. Конечное число нетривиальных показателей $\left\{\left(n_{1}, r_{1}\right), \ldots,\left(n_{s}, r_{s}\right)\right\}, n_{i}>1$, назовем сигнатурой $G$-пучка $L$ и обозначим через $\sigma(L)$.

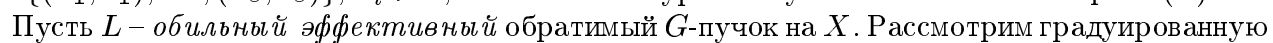
алгебру $A(L)=\bigoplus_{n \geqslant 0} \Gamma\left(L^{\otimes n}\right)$ голоморфных сечений, ассоциированную с пучком $L$, и ее градуированную подалгебру $G$-инвариантных сечений $A(L)^{G}$.

При каких условиях алгебра $A(L)^{G}$ свободна, т.е. изоморфна градуированной алгебре многочленов $\mathbb{C}\left[T_{1}, T_{2}\right]$ ? Ответ на этот вопрос будет дан в терминах степени $\operatorname{deg} L$ пучка $L$ на $X$, порядка $|G|$ группы $G$ и сигнатуры $\sigma\left(L^{\vee}\right)=\left\{\left(n_{1}, e_{1}\right),\left(n_{2}, e_{2}\right), \ldots,\left(n_{s}, e_{s}\right)\right\}$ двойственного обратимого $G$-пучка $L^{\vee}$. Заметим, что сигнатура и отношение $\operatorname{deg} L /|G|$ определяют $G$-пучок $L$ однозначно, с точностью до изоморфизма.

\section{2. Формулировка результата.}

Теорема. Алгебра $A(L)^{G}$ тогда и только тогда свободна, когда выполняется одно из трех (взаимоисключающих) условий:

1) $\sigma\left(L^{\vee}\right)=\left\{\left(n_{1}, 0\right), \ldots,\left(n_{s}, 0\right)\right\} u \operatorname{deg} L /|G|=1$;

2) $\sigma\left(L^{\vee}\right)=\left\{\left(n_{1}, n_{1}-1\right),\left(n_{2}, 0\right), \ldots,\left(n_{s}, 0\right)\right\} u \operatorname{deg} L /|G|=\frac{1}{n_{1}}$;

3) $\sigma\left(L^{\vee}\right)=\left\{\left(n_{1}, e_{1}\right),\left(n_{2}, e_{2}\right),\left(n_{3}, 0\right), \ldots,\left(n_{s}, 0\right)\right\}$, $\operatorname{deg} L /|G|=\frac{1}{n_{1} n_{2}} u \frac{1}{n_{1} n_{2}}+\frac{e_{1}}{n_{1}}+\frac{e_{2}}{n_{2}}=1$.

В первом случае $A(L)^{G}=\mathbb{C}\left[T_{1}, T_{2}\right], \operatorname{deg} T_{1}=1, \operatorname{deg} T_{2}=1$, а во втором и третвем $\operatorname{deg} T_{1}=n_{1}, \operatorname{deg} T_{2}=1 u \operatorname{deg} T_{1}=n_{1}, \operatorname{deg} T_{2}=n_{2}$ соответственно.

3. Набросок доказательства теоремы. Докажем необходимость условий теоремы. Достаточность следует из обратимости ключевых лемм. Напомним конструкцию Брискорна-Долгачева ([1]-[3]): вложение $X \hookrightarrow L^{\vee}$ в качестве нулевого сечения определяет вложение рациональной кривой $C=X / G$ в нормальную поверхность $Y=L^{\vee} / G$. Кривая $C$ стягивается на поверхности $Y$ в точку, рождая нормальную особенность $\left(Y, y_{0}\right)$ Брискорна-Долгачева. Пусть $I$ - множество тех индексов $k$, для которых $e_{k}>0$ (можно считать, что $I=\{1,2, \ldots, t\}, t \leqslant s$ ). Граф Г дивизора хорошего разрешения этой особенности имеет вид звезды

$$
\left[n_{1}, e_{1}\right]
$$

у которой центральная рациональная кривая имеет индекс самопересечения $-a, a=\operatorname{deg} L /|G|+$ $\sum_{i=1}^{t} e_{i} / n_{i}$, а каждый луч $\left[n_{i}, e_{i}\right], i=1, \ldots, t$, представляет из себя линейный графф 


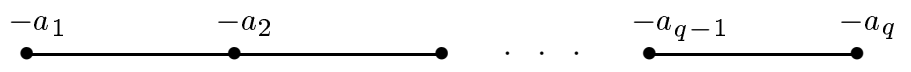

где $a_{k}$ - это компоненты непрерывной дроби вида

$$
n_{i} / e_{i}=a_{1}-\frac{1}{a_{2}-\frac{1}{a_{3}-\ldots}}, \quad a_{i}>1, \quad[4],[2] .
$$

Лемма 1. Алгебра $A(L)^{G}$ тогда и только тогда свободна, когда дивизор разрешения по Брискорну-Долгачеву стягивается в неособую точку.

Утверждение леммы - это прямое следствие определений и стандартных результатов из [1], [3], [4].

ЛЕмма 2. $|I|<3$.

ДокАЗАтЕЛЬСтво. Можно считать, что $s \geqslant 3$. По условию точка $\left(Y, y_{0}\right)$ неособа, в частности, ее локальная фундаментальная группа тривиална. Но, как доказано в [5; теорема 2.2], для этого необходимо, чтобы группа $G$, действующая в $L^{\vee}-X$, порождалась отражениями или, что то же самое, группа $G$ порождалась стабилизаторами $G_{x}$ точек $x \in X$ с показателем $\left(n_{x}, 0\right), n_{x}>1$. Приведем эквивалентную формулировку этого условия в терминах плоской группы $\widetilde{G}$ сигнатуры $\left(0 ; n_{1}, \ldots, n_{s}\right)$ и такой ее нормальной подгруппы без кручения $N$, что $\widetilde{G} / N=G[6]$. Группу $\widetilde{G}$ можно задать образующими $\gamma_{1}, \ldots, \gamma_{s}$ с соотношениями $\prod_{i=1}^{s} \gamma_{i}=1$, $\gamma_{j}^{n_{j}}=1, j=1, \ldots, s$, а условие состоит в том, что $\widetilde{G}$ нормально порождается элементами $\gamma_{t+1}, \ldots, \gamma_{s}$ (напомним, что $I=\{1, \ldots, t\}$ ). Такое возможно, лишь если $|I|<3$. В противном случае существует естественный гомоморфизм группы $\widetilde{G}$ в нетривиальную плоскую группу $\left\langle\gamma_{1}, \ldots, \gamma_{t} \mid \gamma_{1} \cdots \gamma_{t}=1, \gamma_{j}^{n_{j}}=1, j=1, \ldots, t\right\rangle$, ядро которого нормально порождено элементами $\gamma_{t+1}, \ldots, \gamma_{s}$.

В силу леммы 2 граф Г линеен, и остается воспользоваться следующим утверждением.

Лемма 3. Дивизор хорошего разрешения по Брискорну-Долгачеву с линейным графом Г тогда и только тогда стягивается в неособую точку, когда

a) $a=1$;

б) определитель матрицы пересечений графа Г равен по модулю единице.

Условия а) и б) леммы 3 эквивалентны диофантовым уравнениям 1), 2), 3) в формулировке теоремы.

Работа выполнена при финансовой поддержке РФФИ. Я благодарю профессора И. Бернштейна за интересные обсуждения, а университеты Тель-Авива и Бар-Илана за финансовую помощь и гостеприимство.

\section{СПИСОК ЛИТЕРАТУРЫ}

[1] Долгачев И. В. // Функц. анализ и его прил. 1975. Т. 9. № 2. С. 67-68. [2] Brieskorn E. // Invent. Math. 1968. V. 4. P. 336-358. [3] Pinkham H. // Math. Ann. 1977. V. 227. Р. 183-193. [4] Долгачев И. В. // Функц. анализ и его прил. 1974. Т. 8. № 2. С. 75-77. [5] Шварцман О.В.// Вопросы теории групп и гомологической алгебры. Ярославль, 1991. С. 32-39. [6] Цишанг Х., Фогт Э., Колдевай Х.- Д. Поверхности и разрывные группы. М.: Наука, 1988. 